PROCEEDINGS OF THE AMERICAN MATHEMATICAL SOCIETY

Volume 124, Number 6, June 1996

\title{
WEAK LAW OF LARGE NUMBERS FOR ALMOST PERIODICALLY CORRELATED PROCESSES
}

\author{
A. MAKAGON AND A. G. MIAMEE
}

(Communicated by Richard Durrett)

\begin{abstract}
This note contains two simple observations concerning the weak law of large numbers for almost periodically correlated processes.
\end{abstract}

Let $H$ be a complex Hilbert space with the inner product $(\cdot, \cdot)$, and let $R$ denote the set of real numbers. Any Borel measurable function $X: R \longrightarrow H$ will be referred to as a stochastic process. A stochastic process $X$ is said to be almost periodically correlated $(A P C)$, if its correlation function $R(s, u)=(X(s), X(u))$ is bounded and uniformly continuous in $s, u$, and for each $t \in R$ the function $B(t, u)=R(t+u, u), u \in R$, is uniformly almost periodic in $u$ [3]. If $X$ is APC, then for every $\lambda, t \in R$ the limit

$$
a_{\lambda}(t)=\lim _{T \rightarrow \infty} \frac{1}{T} \int_{0}^{T} B(t, u) \exp (-i \lambda u) d u
$$

exists, the set $\Lambda=\left\{\lambda \in R: a_{\lambda}(t) \neq 0\right.$ for some $\left.t\right\}$ is countable, and for every $\lambda \in \Lambda$ there is a complex measure $\Gamma_{\lambda}$ (sometimes called the spectral measure corresponding to $a_{\lambda}$ ) such that $a_{\lambda}(t)=\int_{-\infty}^{\infty} \exp (i t x) \Gamma_{\lambda}(d x)$ (see e.g. [4]). An APC process $X$ is called almost periodically unitary $(A P U)$ if there is a continuous unitary group $U(t), t \in R$, and an $H$-valued uniformly almost periodic function $f$ such that $X(t)=$ $U(t) f(t), t \in R[5]$. An APC process X is called uniformly almost periodically correlated $(U A P C)$ if the function $u \longrightarrow B(\cdot, u)$ is uniformly almost periodic from $R$ to $C(R)$, the Banach space of bounded continuous functions on $R$ equipped with the sup-norm [2].

We will say that a process $X$ satisfies the weak law of large numbers (WLLN) if the limit

$$
\lim _{T \rightarrow \infty} \frac{1}{T} \int_{0}^{T} X(t) d t
$$

exists in the norm topology of $H$.

The problem of existence of the limit (2) for APC processes was raised in [1], where among other results it was proved that if $X$ is APC and $\sum_{\lambda \in \Lambda-\{0\}} \lambda^{-2}$ is finite, then $X$ satisfies WLLN. The purpose of this note is to show that for APC processes WLLN holds when the set $\Lambda-\{0\}$ is separated from zero. This result

Received by the editors November 15, 1994.

1991 Mathematics Subject Classification. Primary 60G12, 60F05.

Key words and phrases. Weak law of large numbers, almost periodically correlated processes.

This research was supported by the Office of Naval Research Grant No. N00014-89-J-1824.

(c)1996 American Mathematical Society 
is more general than the one presented in [1]; but our technique of the proof is quite similar. We also observe that every UAPC process satisfies WLLN, which generalizes the known fact that any APU process satisfies WLLN. However, we need to admit that it is not known whether the inclusions $A P U \subseteq U A P C \subseteq A P C$ are proper [5].

The proofs of both results are based on the following simple lemma, which summarizes the method used in [1].

Lemma 1. A locally integrable stochastic process $X$ with the correlation function $R(s, u)$ satisfies $W L L N$ if and only if for every $\varepsilon>0$ there exist functions $R_{\varepsilon}(s, u)$ and $E_{\varepsilon}(s, u)$, and a finite set $\Lambda_{\varepsilon}$ with $0 \notin \Lambda_{\varepsilon}$, such that:

(i): $R_{\varepsilon}(t+u, u)=r_{0}(t)+\sum_{\lambda \in \Lambda_{\varepsilon}} r_{\lambda}(t) \exp (i \lambda u)$, where for $\lambda \in \Lambda_{\varepsilon} \cup\{0\}$ the functions $r_{\lambda}$ are bounded and measurable, and $r_{0}(\cdot)$ is such that the limit $\lim _{A, B \rightarrow \infty} \frac{1}{A B} \int_{0}^{A} \int_{0}^{B} r_{0}(s-t) d s d t$ exists,

(ii): $\left|\frac{1}{A B} \int_{0}^{A} \int_{0}^{B} E_{\varepsilon}(s, u) d s d u\right|<\varepsilon$, for large $A$ and $B$,

(iii): $R(s, u)=R_{\varepsilon}(s, u)+E_{\varepsilon}(s, u), s, u \in R$.

Proof. Let us denote $\mathcal{M}_{A, B}(g)=\frac{1}{A B} \int_{0}^{A} \int_{0}^{B} g(s, u) d s d u$. It is clear that the limit (2) exists iff $\mathcal{M}_{A, B}(R)$ is Cauchy as $A, B \rightarrow \infty$, that is, if for every $\varepsilon>0$ there is $M>0$ such that if $A, B, C, D>M$, then $\left|\mathcal{M}_{A, B}(R)-\mathcal{M}_{C, D}(R)\right|<\varepsilon$. Suppose first that $R(s, u)=R_{\varepsilon}(s, u)+E_{\varepsilon}(s, u), s, u \in R$, where $R_{\varepsilon}$ and $E_{\varepsilon}$ satisfy (i) - (iii). First we observe that $\mathcal{M}_{A, B}\left(R_{\varepsilon}\right)$ converges to zero as $A, B \rightarrow \infty$. Because $\Lambda_{\varepsilon}$ is finite, it suffices to show that $\mathcal{M}_{A, B}\left(r_{\lambda}(s-u) e^{i \lambda u}\right) \rightarrow 0$, as $A, B \rightarrow \infty$ for every bounded measurable function $r_{\lambda}(t)$ and a non-zero $\lambda$. Substituting $s=u+t$ in the above integral and changing the order of integration we obtain that

$$
\begin{aligned}
\left|\mathcal{M}_{A, B}\left(r_{\lambda}(s-u) e^{i \lambda u}\right)\right| & =\left|\frac{1}{A B} \int_{-A}^{B} \frac{\exp (i \lambda b(t))-\exp (i \lambda a(t))}{i \lambda} r_{\lambda}(t) d t\right| \\
& \leq \frac{A+B}{A B} \frac{2}{|\lambda|} \sup _{t}\left|r_{\lambda}(t)\right| \stackrel{A, B \rightarrow \infty}{\longrightarrow} 0,
\end{aligned}
$$

with proper functions $a(t)$ and $b(t)$. Consequently $\left|\mathcal{M}_{A, B}\left(R_{\varepsilon}\right)-\mathcal{M}_{C, D}\left(R_{\varepsilon}\right)\right|<\varepsilon$ for large $A, B, C, D$, and from (ii) and (iii) it follows that $\left|\mathcal{M}_{A, B}(R)-\mathcal{M}_{C, D}(R)\right|<3 \varepsilon$ for large $A, B, C, D$.

Conversely, suppose that the $\operatorname{limit} \zeta=\lim _{T \rightarrow \infty}(1 / T) \int_{0}^{T} X(t) d t$ exists. Let $\varepsilon>0$. Take $\Lambda_{\varepsilon}=\phi$ (the empty set) and $r_{0}(t)=\|\zeta\|^{2}$. Then $R_{\varepsilon}(s, u)=\|\zeta\|^{2}$ and $E_{\varepsilon}(s, u)=R(s, u)-\|\zeta\|^{2}$. Since $\lim _{A, B \rightarrow \infty} \frac{1}{A B} \int_{0}^{A} \int_{0}^{B} r_{0}(s-t) d s d t=\|\zeta\|^{2}$, condition (i) is satisfied. Moreover,

$$
\frac{1}{A B} \int_{0}^{A} \int_{0}^{B} E_{\varepsilon}(s, u) d s d u=\left(\frac{1}{A} \int_{0}^{A} X(s) d s, \frac{1}{B} \int_{0}^{B} X(u) d u\right)-\|\zeta\|^{2} \stackrel{A, B \rightarrow \infty}{\longrightarrow} 0
$$

and hence (ii) also holds true.

Remark 1. Note that $X$ is not assumed to be APC, and $R_{\varepsilon}, E_{\varepsilon}$ or $r_{0}$ do not need to be positive definite.

Corollary 1. Let $X$ be APC and let $a_{\lambda}(t)$ and $\Lambda$ be defined as in (1). If $\Lambda-\{0\}$ is separated from zero, then $X$ satisfies WLLN. 
Proof. From ( $[10], 6.3$ Thm. 3 ) it follows that there is a constant $M<\infty$, independent of $t$ (the latter is clear from proof of the cited theorem), such that for every $t \in R$ and $b>0$

$$
\left|\int_{0}^{b}\left(B(t, u)-a_{0}(t)\right) d u\right| \leq M
$$

Taking $R_{\varepsilon}(s, u)=a_{0}(s-u)$ and $E_{\varepsilon}(s, u)=R(s, u)-a_{0}(s-u)$, and substituting $s=t+u$ in the first integral below, we obtain that for large $A, B$

$$
\begin{aligned}
\left|\frac{1}{A B} \int_{0}^{A} \int_{0}^{B} E_{\varepsilon}(s, u) d s d u\right| & \leq \frac{1}{A B} \int_{-A}^{B}\left|\int_{\phi(t)}^{\psi(t)}\left(B(t, u)-a_{0}(t)\right) d u\right| d t \\
& \leq \frac{A+B}{A B} 2 M \leq \varepsilon .
\end{aligned}
$$

Corollary 2. Let $X$ be UAPC. Then $X$ satisfies WLLN.

Proof. Fix $\varepsilon>0$. Since $X$ is UAPC, there is a polynomial

$$
p(\cdot, u)=p_{0}(\cdot)+\sum_{\lambda \in \Lambda_{\varepsilon}} p_{\lambda}(\cdot) \exp (i \lambda u),
$$

where $\Lambda_{\varepsilon}$ is finite and $0 \notin \Lambda_{\varepsilon}$, such that $\sup _{t} \sup _{u}|B(t, u)-p(t, u)|<\varepsilon / 2$. Let $R_{\varepsilon}(t+u, u)=p(t, u)-p_{0}(t)+a_{0}(t)=a_{0}(t)+\sum_{\lambda \in \Lambda_{\varepsilon}} p_{\lambda}(t) \exp (i \lambda u)$ and $E_{\varepsilon}(s, u)=$ $R(s, u)-R_{\varepsilon}(s, u)$. Then $R_{\varepsilon}(s, u)$ satisfies (i) of Lemma 1. Moreover for all $t, u \in R$

$$
\left|E_{\varepsilon}(t+u, u)\right| \leq|B(t, u)-p(t, u)|+\left|p_{0}(t)-a_{0}(t)\right| \leq \varepsilon
$$

for $\left|p_{0}(t)-a_{0}(t)\right| \leq \lim _{T \rightarrow \infty} \frac{1}{T} \int_{0}^{T}|B(t, u)-p(t, u)| d u \leq \varepsilon / 2$. Therefore (ii) of Lemma 1 is satisfied.

Remark 2. Note that by Lyusternik's theorem ([10], 1.3), an APC process is UAPC if and only if the set $\{B(t, \cdot): t \in R\}$ is relatively compact in $A P(R)$, the space of uniformly almost periodic functions with sup-norm. Therefore, Corollary 2 states that if $\{B(t, \cdot): t \in R\}$ is relatively compact in $A P(R)$, then WLLN holds.

Remark 3. In [1], in addition to (2), the limits

$$
\zeta_{\mu}=\lim _{T \rightarrow \infty} \frac{1}{T} \int_{0}^{T} X(t) \exp (-i \mu t) d t, \mu \in R,
$$

were also studied. Observe that if $X$ is APC, then for each fixed $\mu \in R$ the process $X_{\mu}(t)=X(t) \exp (-i \mu t)$ is also APC, and $B_{\mu}(t, u)=\left(X_{\mu}(t+u), X_{\mu}(u)\right)=$ $\exp (-i \mu t) B(t, u)$. Hence $X$ is UAPC iff $X_{\mu}$ is, and both $X$ and $X_{\mu}$ have the same set of nonzero Fourier coefficients $\Lambda$. Therefore, under the assumptions of Corollary 1 or Corollary 2, all the limits $\zeta_{\mu}, \mu \in R$, also exist.

Limit results for APC and PC processes have also been studied under the socalled $\phi$-mixing condition (see e.g. [6], [7], [8], [9]), which in most cases yields almost sure convergence of the averages. The property of being $\phi$-mixing is of probabilistic type and, at least for Gaussian processes, it seems to be related to the behavior of the function $B(t, u)$ for large $t$ rather than to the distribution of the set $\Lambda$. The relationship between these two types of conditions is not clear at this moment (see also the discussion in [6], Section 4 ) and is worth being studied. We would like to thank the referee of the paper for pointing out to us a possible connection between the two approaches and for other valuable remarks. 


\section{REFERENCES}

1. Cambanis, S. C., Houdre, C. L., Hurd, H. L., Leskow, J. (1994). Laws of Large Numbers for Periodically and Almost Periodically Correlated Processes, Stoch. Processes and their Appl. 53, 37-54. CMP 94:17

2. Dehay, D. Spectral Analysis of the Covariance Kernel of the of Almost Periodically Correlated Processes. Preprint.

3. Gladyshev, E. (1963). Periodically and almost periodically correlated random processes with continuous time. Th. Probab.Appl. 8, 173-177. MR 27:1986

4. Hurd, H. L. (1991). Correlation Theory of Almost Periodically Correlated Processes. J. Mult. Analysis 37, 24-45. MR 92e:60074

5. Hurd, H. L. (1992). Almost Periodically Unitary Stochastic Processes. Stoch. Processes and their Appl. 43, 99-113. MR 94g:60068

6. Hurd, H. L., Leskow, J. (1992). Estimation of the Fourier coefficients and their spectral densities for $\phi$-mixing almost periodically correlated processes. Statistics and Prob. Letters 14, 299-306. MR 93h:62172

7. Hurd, H. L., Leskow, J. (1992). Strongly consistent and asymptotically normal estimation of the covarianvce for almost periodically correlated stochastic processes. Statistics and Decision 10, 201-225. MR 94d:62213

8. Leskow, J. (1994). Asymptotic normality of the spectral density estimator for almost periodically correlated stochastic processes. Stoch. Processes and their Appl. 52, 351-360. CMP 94:17

9. Leskow J., Weron, A. (1992). Ergodic behavior and estimation for periodically correlated processes. Statistics and Prob. Letters 15, 299-304. MR 94k:62147

10. Levitan, B. M., Zhikov, V. V. Almost periodic functions and differential equations. Cambridge Univ. Press. 1982. MR 84g:34004

Department of Mathematics, Hampton University, Hampton, Virginia 26668

E-mail address: miamee@cs.hamptonu.edu

E-mail address: makagon@math1.math.hamptonu.edu 